\title{
INVERSE STOCHASTIC DOMINANCE, MAJORIZATION, AND MEAN ORDER STATISTICS
}

\author{
JESÚS DE LA CAL, ${ }^{*}$ Universidad del País Vasco \\ JAVIER CÁRCAMO, ${ }^{* *}$ Universidad Autónoma de Madrid
}

\begin{abstract}
The inverse stochastic dominance of degree $r$ is a stochastic order of interest in several branches of economics. We discuss it in depth, the central point being the characterization in terms of the weak $r$-majorization of the vectors of expected order statistics. The weak $r$-majorization (a notion introduced in the paper) is a natural extension of the classical (reverse) weak majorization of Hardy, Littlewood and Pòlya. This work also shows the equivalence between the continuous majorization (of higher order) and the discrete $r$-majorization. In particular, our results make it clear that the cases $r=1,2$ differ substantially from those with $r \geq 3$, a fact observed earlier by Muliere and Scarsini (1989), among other authors. Motivated by this fact, we introduce new stochastic orderings, as well as new social inequality indices to compare the distribution of the wealth in two populations, which could be considered as natural extensions of the first two dominance rules and the S-Gini indices, respectively.
\end{abstract}

Keywords: Stochastic orders; inverse stochastic dominance; order statistic; majorization; S-Gini index; inequality measurement

2000 Mathematics Subject Classification: Primary 60E15; 60E99; 26D15

\section{Introduction: $r$-inverse stochastic dominance}

Stochastic orderings have significant applications in many scientific areas. For example, several stochastic orders are used in economics to compare risks under uncertainty (see Denuit et al. (2005)). The distribution of the wealth in different populations or years has also been analyzed using Lorenz curves and Gini indices which are closely related to stochastic orders (see Lambert (1993)). Moreover, in reliability theory, stochastic orders are extremely important to compare lifetime distributions or define classes of ageing distributions (see Lai and Xie (2006)). In this paper we consider some order relations based on the comparison of the quantile functions of the random variables.

Throughout this paper, the notation $(X, F)$ means that $X$ is an integrable random variable having distribution function $F$ (only integrable random variables will be considered), and $F_{r}^{-1}$ $(r \geq 1)$ stands for the $r$-quantile function of $X$, which is recursively defined by

$$
\begin{gathered}
F_{1}^{-1}(t):=\inf \{x: F(x) \geq t\}, \quad 0<t<1, \\
F_{r}^{-1}(t):=\int_{0}^{t} F_{r-1}^{-1}(y) \mathrm{d} y, \quad 0 \leq t \leq 1, r \geq 2 .
\end{gathered}
$$

Received 6 July 2009; revision received 11 November 2009.

* Postal address: Departamento de Matemática Aplicada y Estadística e Investigación Operativa, Facultad de Ciencia y Tecnología, Universidad del País Vasco, Apartado 644, 48080 Bilbao, Spain. Email address: jesus.delacal@ehu.es

** Postal address: Departamento de Matemáticas, Facultad de Ciencias, Universidad Autónoma de Madrid, 28049 Madrid, Spain. Email address: javier.carcamo@uam.es 
Observe that $F_{1}^{-1}$ is the usual quantile function of $X$. Also, the integrability of $X$ guarantees that the preceding definition is consistent, because it is readily shown (by induction on $r$ ) that

$$
\left|F_{r}^{-1}(t)\right| \leq \frac{t^{r-2}}{(r-2) !} \mathrm{E}|X|, \quad 0<t<1, r \geq 2
$$

(where $\mathrm{E}$ is the expectation operator), and, therefore, $F_{r}^{-1}$ is integrable on $(0,1)$ for all $r \geq 1$.

By means of these functions, we can define a sequence of partial orders in the set of the probability distributions with finite expectation.

Definition 1. Given $(X, F),(Y, G)$, and $r \geq 1$, we write $X \leq_{r} Y$ if

$$
F_{r}^{-1}(t) \leq G_{r}^{-1}(t), \quad 0<t<1 .
$$

It is clear that $X \leq_{r} Y$ implies that $X \leq_{r+1} Y(r \geq 1)$. Also, ' $\leq_{1}$ ' is just the usual stochastic order, and ' $\leq 2$ ' coincides with the increasing concave order (see Müller and Stoyan (2002) and Shaked and Shanthikumar (2006)). Thus ' $\leq_{r}$ ' can be viewed as an extension of ' $\leq 1$ ' and ' $\leq 2$ '.

These orderings are of interest in several branches of economics, where ' $\leq 1$ ' and ' $\leq 2$ ' are usually called the first- and second-order stochastic dominances, respectively, and ' $\leq_{r}$ ' is known as the inverse stochastic dominance of degree $r$ (abbreviated $r$-ISD). For $r \geq 3$, they were introduced in Muliere and Scarsini (1989) to analyze the social inequality, and they have also been considered in Aaberge (2009), Dentcheva and Ruszczynski (2006), Maccheroni et al. (2005), and Zoli (1999), (2002), among other works.

The stochastic orders ' $\leq_{r}$ ' $(r \geq 2)$ compare the lowest part of the distributions, since we are recursively integrating the quantile function over the interval $(0, t)$. For this reason, these relations are usually considered in the literature of social inequality and welfare measurement where the strata in the populations with less resources is of major interest (see Aaberge (2009) and the references therein).

If, however, we are interested in the right tail of the distributions, we can change the interval of integration in $(1)$ to $(t, 1)$. For instance, in actuarial sciences the risk associated to large-loss events is exceedingly important. Therefore, in this area the considered orders are defined in a like manner, but the integration is over $(t, 1)$. For $r=2$, the relation generated in this way is called the stop loss order in actuarial sciences and the increasing convex order in probability theory. See Denuit et al. (2005) for more details about the use of these stochastic orders in risk analysis. Analogous orderings (defined by using the inverse of the survival function instead of the quantile function) have been discussed in Wang and Young (1998); they were called dual stochastic dominance by the authors, since they are closely related to Yaari's dual theory of choice under risk (see Yaari (1987)). It is clear that all the results obtained when we integrate over $(0, t)$ can be immediately translated to the case in which the interval of integration is $(t, 1)$. Hence, from now on we just consider the orderings given in Definition 1.

The comparison of the $r$ th primitives of the distribution functions (instead of the quantile functions) leads to the so-called (direct) stochastic dominance of degree $r$ (abbreviated $r$-SD); see, for instance, Fishburn (1976) and Levy (1992). This approach has been widely used, and it is closely connected with the $r$-(increasing) concave orderings (see Denuit et al. (1998), (2000)). However, an important drawback of the $r$-SD is that it requires the variables to have a finite $(r-1)$ th moment, and many important distributions do not fulfill this integrability condition. 
From the mathematical analysis point of view, $X \leq_{2} Y$ is equivalent to the (reverse) weak continuous majorization between the quantile functions of $X$ and $Y$ (see Marshall and Olkin (1979)). Thus, relation ' $\leq_{r}$ ' can be viewed as a generalization of the reverse continuous weak majorization.

In the present paper, which follows the spirit of de la Cal and Cárcamo (2006), we focus attention on the characterization of the $r$-ISD in terms of the weak $r$-majorization of the vectors of mean order statistics. The concept of weak $r$-majorization can be viewed as an extension, considered here for the first time, of the classical notion of (reverse) weak majorization introduced by Hardy, Littlewood and Pòlya. Apart from the mathematical interest of these connections between probability, analysis, and economics, various benefits concerning applications could also be expected from the main ideas in this paper: a better understanding of the dominance rules under consideration; the construction of new social inequality indices relevant to the inverse stochastic dominance; and the theoretical basis to generate hypotheses tests related to stochastic dominance assumptions similar to those considered in Berrendero and Cárcamo (2009), (2010) and Baíllo et al. (2009). However, this empirical counterpart is not explored here.

The paper is organized as follows. In the next section we discuss the notion of weak $r$-majorization of $n$-dimensional vectors. Section 3 collects the necessary auxiliary tools to achieve our main results in Section 4 concerning the characterization of the $r$-ISD in terms of the weak $r$-majorization of the vectors of mean order statistics. Motivated by such results, in Sections 5 and 6, we introduce and discuss two new stochastic orders, named positive r-ISD and strong $r$-ISD, respectively. Finally, in the last section we introduce and discuss a generalization of the S-Gini indices based on the previous results.

\section{Weak $r$-majorization of vectors}

Let $\boldsymbol{x}:=\left(x_{1}, \ldots, x_{n}\right) \in \mathbb{R}^{n}$. For $1 \leq k \leq n$, the $r$-cumulative sums of $\boldsymbol{x}$ of order $k$ are recursively defined by

$$
s_{k: n}^{[1]}(\boldsymbol{x}):=x_{k: n}, \quad s_{k: n}^{[r]}(\boldsymbol{x}):=\sum_{i=1}^{k} s_{i: n}^{[r-1]}(\boldsymbol{x}), \quad r \geq 2,
$$

where $x_{1: n} \leq x_{2: n} \leq \cdots \leq x_{n: n}$ are the components of $\boldsymbol{x}$ arranged in increasing order. We actually have

$$
s_{k: n}^{[r]}(\boldsymbol{x})=\sum_{i=1}^{k}\left(\begin{array}{c}
k-i+r-2 \\
r-2
\end{array}\right) x_{i: n}, \quad 1 \leq k \leq n, r \geq 2,
$$

as it readily follows by induction on $r$, interchanging the order of summation, and applying the following elementary property of the binomial coefficients:

$$
\sum_{j=i}^{k}\left(\begin{array}{c}
j-i+m-1 \\
m-1
\end{array}\right)=\left(\begin{array}{c}
k-i+m \\
m
\end{array}\right), \quad m=1,2, \ldots
$$

Definition 2. For $\boldsymbol{x}, \boldsymbol{y} \in \mathbb{R}^{n}$ and $r \geq 1$, we say that $\boldsymbol{x}$ is weakly $r$-majorized by $\boldsymbol{y}$, written $\boldsymbol{x} \prec_{r} \boldsymbol{y}$, if

$$
s_{k: n}^{[r]}(\boldsymbol{x}) \leq s_{k: n}^{[r]}(\boldsymbol{y}), \quad 1 \leq k \leq n .
$$


Remark 1. It is clear that $\boldsymbol{x} \prec_{r} \boldsymbol{y}$ implies that $\boldsymbol{x} \prec_{r+1} \boldsymbol{y}$. The relations ' $\prec_{r}$ ' are preorders in $\mathbb{R}^{n}$, but they are (partial) orders in the set $\left\{\boldsymbol{x}=\left(x_{1}, \ldots, x_{n}\right) \in \mathbb{R}^{n}: x_{1} \leq \cdots \leq x_{n}\right\}$. Here ' $\prec_{1}$ ' is nothing but the componentwise order of the increasing rearrangements of the vectors, while ' $\prec_{2}$ ' coincides with the reverse weak majorization, that is, in the terminology and notation of Marshall and Olkin (1979, p. 10), $\boldsymbol{x} \prec 2 \quad \boldsymbol{y}$ is just another way of writing $\boldsymbol{y} \prec^{w} \boldsymbol{x}$. To the best of the authors' knowledge, relations ' $\prec_{r}$ ', with $r \geq 3$, are considered here for the first time.

Remark 2. If the components of the vector $\boldsymbol{x}$ represent the income of the individuals in the population of size $n$ given by $\boldsymbol{x}, s_{k: n}^{[1]}(\boldsymbol{x})$ is the income of the $k$ th poorest individual, $s_{k: n}^{[2]}(\boldsymbol{x})$ is the income sum of the $k$ poorest individuals in the population, and so on. As $r$ increases, the function $s_{k: n}^{[r]}$ places more and more weight on the lowest components. Therefore, the relation $\boldsymbol{x} \prec_{r} \boldsymbol{y}$ means that the distribution of the income in $\boldsymbol{y}$ is more even than in $\boldsymbol{x}$, in the sense that the poorest individuals in $\boldsymbol{y}$ accumulate more proportional and well-balanced income than in $\boldsymbol{x}$. In particular, given any vector $\boldsymbol{x}:=\left(x_{1}, \ldots, x_{n}\right) \in \mathbb{R}^{n}$, it is readily checked that $\boldsymbol{x} \prec_{r} \overline{\boldsymbol{x}}(r \geq 2)$, where $\overline{\boldsymbol{x}}:=(\bar{x}, \ldots, \bar{x}) \in \mathbb{R}^{n}$, with $\bar{x}:=(1 / n) \sum_{i=1}^{n} x_{i}$. Thus, the most equitable vector (with respect to the weak $r$-majorization) is the constant vector of means.

Remark 3. Let $\boldsymbol{x}, \boldsymbol{y} \in \mathbb{R}^{n}$, and denote by $X$ and $Y$ the (discrete) random variables having uniform distribution on the components of $\boldsymbol{x}$ and $\boldsymbol{y}$, respectively. It can be checked that, for $r=1,2$,

$$
\boldsymbol{x} \prec_{r} \boldsymbol{y} \text { if and only if } X \leq_{r} Y
$$

(an indirect proof, in the case $r=2$, can be found in de la Cal and Cárcamo (2006, Remark 2.3)). However, the preceding equivalence is no longer true for $r \geq 3$. For example, when $\boldsymbol{x}=(1,2)$ and $\boldsymbol{y}=(1.1,1.75)$, we have $\boldsymbol{x} \nprec_{3} \boldsymbol{y}$ and $X \leq_{3} Y$. This fact makes it clear that there are subtle differences between the cases $r=1,2$ and $r \geq 3$. This will become apparent in the following sections.

\section{Auxiliary tools and results}

In what follows, given $(X, F)$ and $n \geq 1$, we use the notation

$$
\boldsymbol{\mu}_{n}^{X}:=\left(\mathrm{E} X_{1: n}, \ldots, \mathrm{E} X_{n: n}\right)
$$

where $X_{i: n}$ the $i$ th order statistic of a random sample of size $n,\left(X_{1}, \ldots, X_{n}\right)$, from $(X, F)$. It is well known (see Arnold et al. (2008, Equation (5.2.8))) that

$$
\mathrm{E} X_{i: n}=\mathrm{E} F_{1}^{-1}\left(\beta_{i, n+1-i}\right), \quad n \geq 1,1 \leq i \leq n,
$$

where, for $i, j>0, \beta_{i, j}$ is a random variable having the $\operatorname{Beta}(i, j)$ density given by

$$
B_{i, j}^{[0]}(t):=\frac{(i+j-1) !}{(i-1) !(j-1) !} t^{i-1}(1-t)^{j-1} 1_{(0,1)}(t),
$$

where, here and hereafter, $1_{A}$ is the indicator function of the set $A$. We use the convention that $\beta_{i, 0}(i>0)$ is degenerate at 1 , and set, for $i>0$ and $j \geq 0$,

$$
\begin{aligned}
B_{i, j}^{[1]}(t) & :=\mathrm{P}\left(\beta_{i, j}>t\right), \quad 0 \leq t \leq 1, \\
B_{i, j}^{[r]}(t) & :=\int_{t}^{1} B_{i, j}^{[r-1]}(y) \mathrm{d} y, \quad 0 \leq t \leq 1, r \geq 2 .
\end{aligned}
$$


Lemma 1. For $r \geq 1, m \geq 1$, and $1 \leq k \leq m-1$, we have

$$
\sum_{i=1}^{k} B_{i, m-i}^{[r-1]}(t)=(m-1) B_{k, m-1-k}^{[r]}(t), \quad 0<t<1 .
$$

Proof. Fix $m \geq 1,1 \leq k \leq m-1$, and $0<t<1$, and proceed by induction on $r$. Since

$$
\begin{aligned}
\sum_{i=1}^{k} B_{i, m-i}^{[0]}(t) & =\sum_{i=1}^{k} \frac{(m-1) !}{(i-1) !(m-i-1) !} t^{i-1}(1-t)^{m-i-1} \\
& =(m-1) \sum_{i=0}^{k-1}\left(\begin{array}{c}
m-2 \\
i
\end{array}\right) t^{i}(1-t)^{m-2-i} \\
& =(m-1) B_{k, m-1-k}^{[1]}(t)
\end{aligned}
$$

(the last equality holds by Johnson et al. (2005, Equation (3.30))), the result holds true when $r=1$. Now, if $r \geq 1$, we have, from (5) and the induction hypothesis,

$$
\begin{aligned}
\sum_{i=1}^{k} B_{i, m-i}^{[r]}(t) & =\sum_{i=1}^{k} \int_{t}^{1} B_{i, m-i}^{[r-1]}(y) \mathrm{d} y \\
& =(m-1) \int_{t}^{1} B_{k, m-1-k}^{[r]}(y) \mathrm{d} y \\
& =(m-1) B_{k, m-1-k}^{[r+1]}(t),
\end{aligned}
$$

completing the proof.

Lemma 2. Let $(X, F)$ be given. We have, for $r \geq 2$ and $1 \leq k \leq n-(r-2)$,

$$
s_{k: n}^{[r]}\left(\boldsymbol{\mu}_{n}^{X}\right)=n \frac{r-1}{\int_{0}^{1}} F_{r-1}^{-1}(t) B_{k, n-(r-2)-k}^{[1]}(t) \mathrm{d} t,
$$

where $n \underline{m}:=n(n-1) \cdots(n-m+1)$. In particular,

$$
s_{n-(r-2): n}^{[r]}\left(\mu_{n}^{X}\right)=n \frac{r-1}{r} F_{r}^{-1}(1)=\left(\begin{array}{c}
n \\
r-1
\end{array}\right) \text { E } X_{1: r-1} .
$$

Proof. The equalities in (7) follow from (6) and the fact that (by repeated integrations by parts)

$$
F_{r}^{-1}(1)=\frac{1}{(r-1) !} \int_{0}^{1} F_{1}^{-1}(t)(1-t)^{r-1} \mathrm{~d} t=\frac{1}{(r-1) !} \text { E } X_{1: r-1} .
$$

To show (6), we proceed by induction on $r$. From (4) and Lemma 1 (with $r=1$ and $m=n+1$ ), we have, for $1 \leq k \leq n$,

$$
\begin{aligned}
s_{k: n}^{[2]}\left(\boldsymbol{\mu}_{n}^{X}\right) & =\sum_{i=1}^{k} \mathrm{E} X_{i: n} \\
& =\int_{0}^{1} F^{-1}(t)\left(\sum_{i=1}^{k} B_{i, n+1-i}^{[0]}(t)\right) \mathrm{d} t \\
& =n \int_{0}^{1} F^{-1}(t) B_{k, n-k}^{[1]}(t) \mathrm{d} t
\end{aligned}
$$


showing that (6) holds true, when $r=2$. Now, if $r \geq 2$ and $1 \leq k \leq n-r+1$, we have, by the induction hypothesis, Lemma 1 (with $r=2$ and $m=n-(r-2)$ ), and Fubini's theorem,

$$
\begin{aligned}
s_{k: n}^{[r+1]}\left(\boldsymbol{\mu}_{n}^{X}\right) & =\sum_{i=1}^{k} s_{i: n}^{[r]}\left(\boldsymbol{\mu}_{n}^{X}\right) \\
& =n \frac{r-1}{} \int_{0}^{1} F_{r-1}^{-1}(t)\left(\sum_{i=1}^{k} B_{i, n-(r-2)-i}^{[1]}(t)\right) \mathrm{d} t \\
& =n^{\underline{r}} \int_{0}^{1} F_{r-1}^{-1}(t) B_{k, n-(r+1-2)-k}^{[2]}(t) \mathrm{d} t \\
& =n^{\underline{r}} \int_{0}^{1} F_{r}^{-1}(t) B_{k, n-(r+1-2)-k}^{[1]}(t) \mathrm{d} t
\end{aligned}
$$

completing the proof of (6).

Lemma 3. Let $u(\cdot)$ and $v(\cdot)$ be two positive functions on $(0, \infty)$, increasing to $\infty$ and such that $v(\cdot)>u(\cdot)$. If $\lim _{t \rightarrow \infty}(u(t) / v(t))=l \in[0,1]$ then $\beta_{u(t), v(t)-u(t)}$ converges in $L_{2}$ to $l$ as $t \rightarrow \infty$.

Proof. We have, for $t>0$,

$$
\mathrm{E}\left[\beta_{u(t), v(t)-u(t)}-l\right]^{2}=\frac{u(t)(v(t)-u(t))}{v^{2}(t)(v(t)+1)}+\left(\frac{u(t)}{v(t)}-l\right)^{2} \rightarrow 0 \quad \text { as } t \rightarrow \infty .
$$

The following lemma will play a crucial role in the proof of the main results in the next section.

Lemma 4. Let $(X, F),(Y, G)$, and $r \geq 1$ be given, and assume that

$$
F_{r}^{-1}\left(\frac{a}{b}\right)-G_{r}^{-1}\left(\frac{a}{b}\right) \neq 0
$$

where $a$ and $b$ are integer numbers such that $0<a<b$ and $a / b$ is a continuity point of $F_{r}^{-1}-G_{r}^{-1}$. Then, there exists an integer $m_{0} \geq 1$ such that

$$
s_{a m+c: b m+c}^{[r]}\left(\boldsymbol{\mu}_{b m+c}^{X}\right)-s_{a m+c: b m+c}^{[r]}\left(\boldsymbol{\mu}_{b m+c}^{Y}\right)
$$

has the same sign as $F_{r}^{-1}(a / b)-G_{r}^{-1}(a / b)$ for all $m \geq m_{0}$ and $c=0,1, \ldots, b-1$.

Proof. We start with the case in which $r=1$. Without loss of generality, we can assume that $F_{1}^{-1}(a / b)-G_{1}^{-1}(a / b)>0$. By the continuity assumption, there exist $\delta, \rho>0$ such that

$$
F_{1}^{-1}(t)-G_{1}^{-1}(t)>\delta, \quad t \in I_{\rho}:=(a / b-\rho, a / b+\rho) \subset(0,1) .
$$

Now, by (4) we have

$$
\mathrm{E} X_{a m+c: b m+c}-\mathrm{E} Y_{a m+c: b m+c}=\int_{0}^{1}\left(F_{1}^{-1}(t)-G_{1}^{-1}(t)\right) B_{a m+c,(b-a) m+1}^{[0]}(t) \mathrm{d} t .
$$

Lemma 3 ensures that $\beta_{a m+c,(b-a) m+1} \rightarrow a / b$ in probability as $m \rightarrow \infty$. By this, and the unimodality of the involved beta densities, there exists an $m_{0}$ such that, for $m \geq m_{0}$,

$$
\int_{I_{\rho}} B_{a m+c,(b-a) m+1}^{[0]}(t) \mathrm{d} t \geq \frac{1}{2}
$$


and

$$
\sup _{(0,1)-I_{\rho}} B_{a m+c,(b-a) m+1}^{[0]}(t) \leq \frac{\delta}{4 \int_{0}^{1}\left|F_{1}^{-1}(t)-G_{1}^{-1}(t)\right| \mathrm{d} t} .
$$

Inequalities (8) and (10)-(11) entail that, for $m \geq m_{0}$, the integral on the right-hand side of (9) is larger than $\delta / 4>0$, and the proof of the lemma in the case in which $r=1$ is complete.

Assume now that $r \geq 2$ (in this case, $F_{r}^{-1}-G_{r}^{-1}$ is a continuous function). By Lemma 2 we have, for all $m$ such that $b m \geq r-1$ and $a m \leq b m-(r-2)$,

$$
\begin{aligned}
& \frac{1}{(b m+c) \frac{r-1}{n}}\left(s_{a m+c: b m+c}^{[r]}\left(\boldsymbol{\mu}_{n}^{X}\right)-s_{a m+c: b m+c}^{[r]}\left(\boldsymbol{\mu}_{n}^{Y}\right)\right) \\
& \quad=\int_{0}^{1}\left(F_{r-1}^{-1}(t)-G_{r-1}^{-1}(t)\right) B_{a m+c,(b-a) m-(r-2)}^{[1]}(t) \mathrm{d} t .
\end{aligned}
$$

From Lemma 3 we obtain

$$
\lim _{m \rightarrow \infty} B_{a m+c,(b-a) m-(r-2)}^{[1]}(t)=1_{(-\infty, a / b]}(t), \quad t \neq a / b, c=0,1, \ldots, b-1 .
$$

Since $F_{r-1}^{-1}-G_{r-1}^{-1}$ is integrable and $B_{a m+c,(b-a) m-(r-2)}^{[1]}$ is bounded by 1 , we obtain, by dominated convergence,

$$
\lim _{m \rightarrow \infty} \int_{0}^{1}\left(F_{r-1}^{-1}(t)-G_{r-1}^{-1}(t)\right) B_{a m+c,(b-a) m-(r-2)}^{[1]}(t) \mathrm{d} t=F_{r}^{-1}\left(\frac{a}{b}\right)-G_{r}^{-1}\left(\frac{a}{b}\right)
$$

for $c=0,1, \ldots, b-1$, and the conclusion follows.

Lemma 5. Let $(X, F)$ be given. For $r \geq 2, n \geq r-1$, and $n-(r-2) \leq k \leq n$, we have

$$
s_{k: n}^{[r]}\left(\boldsymbol{\mu}_{n}^{X}\right)=\sum_{i=n+1-k}^{r-1}\left(\begin{array}{l}
n \\
i
\end{array}\right)\left(\begin{array}{c}
r-2-(n-k) \\
r-1-i
\end{array}\right) \text { E } X_{1: i} .
$$

Proof. We use induction on $r \geq 2$. For $r=2$ (this forces $k$ to be $n$ ), equality (12) simply states that $s_{n: n}^{[2]}\left(\boldsymbol{\mu}_{n}^{X}\right)=n \mathrm{E} X$, which trivially holds. Assume that (12) holds true for some $r \geq 2$, and let $n-(r-1) \leq k \leq n$. Since

$$
s_{k: n}^{[r+1]}\left(\boldsymbol{\mu}_{n}^{X}\right)=s_{n-r+1: n}^{[r+1]}\left(\boldsymbol{\mu}_{n}^{X}\right)+\sum_{i=n-r+2}^{k} s_{i: n}^{[r]}\left(\boldsymbol{\mu}_{n}^{X}\right),
$$

we obtain, by (7), the induction hypothesis, and an interchange of the order of summation,

$$
\begin{aligned}
s_{k: n}^{[r+1]}\left(\boldsymbol{\mu}_{n}^{X}\right) & =\left(\begin{array}{l}
n \\
r
\end{array}\right) \mathrm{E} X_{1: r}+\sum_{i=n-r+2}^{k} s_{i: n}^{[r]}\left(\mu_{n}^{X}\right) \\
& =\left(\begin{array}{l}
n \\
r
\end{array}\right) \mathrm{E} X_{1: r}+\sum_{i=n-r+2}^{k} \sum_{j=n+1-i}^{r-1}\left(\begin{array}{c}
n \\
j
\end{array}\right)\left(\begin{array}{c}
r-2-(n-i) \\
r-1-j
\end{array}\right) \mathrm{E} X_{1: j} \\
& =\left(\begin{array}{c}
n \\
r
\end{array}\right) \mathrm{E} X_{1: r}+\sum_{j=n+1-k}^{r-1}\left(\begin{array}{c}
n \\
j
\end{array}\right) \mathrm{E} X_{1: j} \sum_{i=n+1-j}^{k}\left(\begin{array}{c}
r-2-(n-i) \\
r-1-j
\end{array}\right) \\
& =\sum_{i=n+1-k}^{r}\left(\begin{array}{c}
n \\
i
\end{array}\right)\left(\begin{array}{c}
r-1-(n-k) \\
r-i
\end{array}\right) \mathrm{E} X_{1: i} .
\end{aligned}
$$




\section{Characterization of the $\boldsymbol{r}$-ISD in terms of mean order statistics}

In this section we show that all the information given by the inverse dominance is contained in the relationships satisfied by the vectors of mean order statistics of the variables, $\boldsymbol{\mu}_{n}^{X}$ and $\boldsymbol{\mu}_{n}^{Y}(n \geq 1)$. These relations are closely connected with the concept of weak $r$-majorization considered in Section 2. The characterization takes a different form, according to whether $r=1,2$, or $r \geq 3$.

Theorem 1. Let $(X, F)$ and $(Y, G)$ be given. For $r=1,2$, the following assertions are mutually equivalent:

(a) $X \leq r Y$;

(b) $\boldsymbol{\mu}_{n}^{X} \prec_{r} \boldsymbol{\mu}_{n}^{Y}$ for all $n \geq 1$;

(c) $\boldsymbol{\mu}_{n}^{X} \prec_{r} \boldsymbol{\mu}_{n}^{Y}$ for infinitely many values of $n$.

Proof. We have, for $1 \leq k \leq n$,

$$
s_{k: n}^{[1]}\left(\mu_{n}^{X}\right)-s_{k: n}^{[1]}\left(\mu_{n}^{Y}\right)=\int_{0}^{1}\left(F_{1}^{-1}(t)-G_{1}^{-1}(t)\right) B_{k, n+1-k}^{[0]}(t) \mathrm{d} t
$$

(by (4)), and, by Lemma 2 ,

$$
s_{k: n}^{[2]}\left(\mu_{n}^{X}\right)-s_{k: n}^{[2]}\left(\mu_{n}^{Y}\right)=n \int_{0}^{1}\left(F_{2}^{-1}(t)-G_{2}^{-1}(t)\right) B_{k, n-k}^{[1]}(t) \mathrm{d} t .
$$

Thus, (a) implies (b). Assertion (b) trivially implies (c). Finally, assume that (a) fails to be true. Then, $F_{r}^{-1}(t)-G_{r}^{-1}(t)>0$ for some rational number $t \in(0,1)$, which can be assumed to be a continuity point of $F_{r}^{-1}-G_{r}^{-1}$, and, by Lemma 4, there exists an integer $n_{0} \geq 1$ such that $\boldsymbol{\mu}_{n}^{X} \nprec_{r} \boldsymbol{\mu}_{n}^{Y}$ for all $n \geq n_{0}$, that is, (c) does not hold either. This shows that (c) implies (a), and completes the proof of the theorem.

Remark 4. For $r=1$, the last theorem states that $X \leq_{1} Y$ if and only if $\mathrm{E} X_{i: n} \leq \mathrm{E} Y_{i: n}$ for $1 \leq i \leq n$ and all $n \geq 1$ or infinitely many $n$ s. The first implication is well known in the literature. However, the other direction is new. Also, an analogous version of the preceding result, in the case in which $r=2$, was first obtained in de la Cal and Cárcamo (2006, Corollary 2.1).

For $r \geq 3$, we have the following result, the proof of which is omitted because it is similar to the one for $r=2$ (with the obvious modifications).

Theorem 2. Let $(X, F)$ and $(Y, G)$ be given. For $r \geq 3$, the following assertions are mutually equivalent:

(i) $X \leq_{r} Y$;

(ii) $s_{k: n}^{[r]}\left(\boldsymbol{\mu}_{n}^{X}\right) \leq s_{k: n}^{[r]}\left(\boldsymbol{\mu}_{n}^{Y}\right)$ for all $n \geq r-1$ and $1 \leq k \leq n-(r-2)$;

(iii) $s_{k: n}^{[r]}\left(\boldsymbol{\mu}_{n}^{X}\right) \leq s_{k: n}^{[r]}\left(\boldsymbol{\mu}_{n}^{Y}\right)$ for infinitely many values of $n \geq r-1$ and all $k$ such that $1 \leq k \leq$ $n-(r-2)$.

Consider assertions (a)-(c) of Theorem 1. From Theorem 2, it is clear that the chain of implications (b) $\Rightarrow(\mathrm{c}) \Rightarrow$ (a) continues to hold when $r \geq 3$. However, in this case, the remaining implications are no longer true, as the following examples show. 
Example 1. Let $X$ and $Y$ be the discrete uniform variables on the components of $(0,1,2)$ and $(0.5,1,1)$, respectively. It is easy to check that $X \leq_{3} Y$, and (by Theorem 2 ) we have $s_{k: n}^{[3]}\left(\boldsymbol{\mu}_{n}^{X}\right) \leq s_{k: n}^{[3]}\left(\boldsymbol{\mu}_{n}^{Y}\right)$ for all $n \geq 2$ and $1 \leq k \leq n-1$. However, taking into account the facts that $\mathrm{E} X=1, \mathrm{E} Y=\frac{5}{6}, \mathrm{E} \bar{X}_{1: 2}=\frac{5}{9}$, and $\mathrm{E} \bar{Y}_{1: 2}=\frac{13}{18}$, and using Lemma 5, we obtain, for $n \geq 1$,

$$
s_{n: n}^{[3]}\left(\boldsymbol{\mu}_{n}^{X}\right)-s_{n: n}^{[3]}\left(\boldsymbol{\mu}_{n}^{Y}\right)=n(\mathrm{E} X-\mathrm{E} Y)+\frac{n(n-1)}{2}\left(\mathrm{E} X_{1: 2}-\mathrm{E} Y_{1: 2}\right)=\frac{n(3-n)}{12} .
$$

Thus, in this example, we have $\boldsymbol{\mu}_{n}^{X} \nprec_{3} \boldsymbol{\mu}_{n}^{Y}$ if $n=1,2$ and $\boldsymbol{\mu}_{n}^{X} \prec_{3} \boldsymbol{\mu}_{n}^{Y}$ for $n \geq 3$.

Example 2. Let $X$ and $Y$ be the discrete uniform random variables on the components of $(0,1,2)$ and $\left(\frac{5}{13}, \frac{10}{13}, \frac{10}{13}\right)$, respectively. We again have $X \leq_{3} Y$. Moreover, E $X=1>\frac{25}{39}=$ $\mathrm{E} Y$ and $\mathrm{E} X_{1: 2}=\frac{5}{9}=\mathrm{E} Y_{1: 2}$. Therefore, using Lemma 5, we have, for all $n \geq 1$,

$$
s_{n: n}^{[3]}\left(\boldsymbol{\mu}_{n}^{X}\right)-s_{n: n}^{[3]}\left(\boldsymbol{\mu}_{n}^{Y}\right)=n(\mathrm{E} X-\mathrm{E} Y)+\frac{n(n-1)}{2}\left(\mathrm{E} X_{1: 2}-\mathrm{E} Y_{1: 2}\right)=\frac{14}{39} n,
$$

implying that $\boldsymbol{\mu}_{n}^{X} \nprec_{3} \boldsymbol{\mu}_{n}^{Y}$ for all $n \geq 1$.

Remark 5. The essential reason why the implication (a) $\Rightarrow$ (c) (of Theorem 1) is no longer true (when $r \geq 3$ ) lies in the strong dependence among the order statistics. Actually, according to Lemma 5, when $k>n-(r-2), s_{k: n}^{[r]}\left(\boldsymbol{\mu}_{n}^{X}\right)$ depends on the values $\mathrm{E} X_{1: i}$ with $n+1-k \leq i \leq r-1$, that is (recall (7)), it depends on the values $F_{i}^{-1}(1)$ with $i<r$, while relation ' $\leq_{r}$ ' only relies on $F_{r}^{-1}$.

On the other hand, the fact that (when $r \geq 3$ ) assertion (c) (of Theorem 1) does not imply (b) is rather surprising, since the sequence of vectors $\left\{\boldsymbol{\mu}_{n}^{X}: n \geq n_{0}\right\}$ determines the distribution of the variable $X$ (see Huang (1989)).

Remark 6. It is apparent from the previous observations that, regarding the $r$-ISD, the cases $r=1,2$ differ from those with $r \geq 3$. In Muliere and Scarsini (1989), the authors emphasized the fact that, when $r=1,2$, the inverse dominance coincides with the direct dominance, but this is no longer true when $r \geq 3$. The difference between the two dominances was also observed in Shorrocks and Foster (1987), in the case $r=3$.

The preceding discussion on the differences between the cases $r=1,2$ and $r \geq 3$ is the main motivation for the contents of the next two sections.

\section{Positive $r$-ISD}

When $X$ is dominated by $Y$ according to the first or the second dominance, the 'total size' of $Y$ is larger than that of $X$, that is, $\mathrm{E} X \leq \mathrm{E} Y$. This fails to be true for higher ISD rules (see the examples in the previous section). In this section we define new stochastic orderings by adding a suitable size condition.

First, we establish the following auxiliary result. Roughly speaking, it says that the largest $m$ such that $F_{m}^{-1}(1) \neq G_{m}^{-1}(1)$ determines the sign of the cumulative sums $s_{k: n}^{[r]}\left(\boldsymbol{\mu}_{n}^{X}\right)-s_{k: n}^{[r]}\left(\boldsymbol{\mu}_{n}^{Y}\right)$ for large $n$ and $k$.

Lemma 6. Let $(X, F)$ and $(Y, G)$ be given, let $r \geq 2$, and set

$$
M:=\left\{j \in\{2, \ldots, r\}: F_{j}^{-1}(1) \neq G_{j}^{-1}(1)\right\} .
$$

(a) If $M=\varnothing$ then $s_{k: n}^{[r]}\left(\boldsymbol{\mu}_{n}^{X}\right)=s_{k: n}^{[r]}\left(\boldsymbol{\mu}_{n}^{Y}\right)$ for all $n \geq 1$ and $n-(r-2) \leq k \leq n$. 
(b) If $M \neq \varnothing$ then $s_{k: n}^{[r]}\left(\boldsymbol{\mu}_{n}^{X}\right)=s_{k: n}^{[r]}\left(\boldsymbol{\mu}_{n}^{Y}\right)$ for all $n \geq 1$ and $n-(r-2) \leq k \leq n+1-m$, where $m:=\max M$. Moreover, there exists an integer $n_{0} \geq 1$ such that $s_{k: n}^{[r]}\left(\boldsymbol{\mu}_{n}^{X}\right)-s_{k: n}^{[r]}\left(\boldsymbol{\mu}_{n}^{Y}\right)$ has the same sign as $F_{m}^{-1}(1)-G_{m}^{-1}(1)$ for all $n \geq n_{0}$ and $n+2-m \leq k \leq n$.

Proof. Fix $r \geq 2$. Part (a) directly follows from (7) and Lemma 5. Now, if $M \neq \varnothing$, and $m:=\max M$, we have

$$
\begin{gathered}
\mathrm{E} X_{1: i}-\mathrm{E} Y_{1: i}=0, \quad i=m, \ldots, r-1, \\
\mathrm{E} X_{1: m-1}-\mathrm{E} Y_{1: m-1} \neq 0 .
\end{gathered}
$$

Equality (13) and Lemma 5 entail $s_{k: n}^{[r]}\left(\boldsymbol{\mu}_{n}^{X}\right)=s_{k: n}^{[r]}\left(\boldsymbol{\mu}_{n}^{Y}\right)$ for $n \geq 1$ and $n-(r-2) \leq k \leq$ $n+1-m$. Also, for $n+2-m \leq k \leq n$, we have

$$
\frac{s_{k: n}^{[r]}\left(\boldsymbol{\mu}_{n}^{X}\right)-s_{k: n}^{[r]}\left(\boldsymbol{\mu}_{n}^{Y}\right)}{C_{n}(m-1, k, r)}=\left(\mathrm{E} X_{1: m-1}-\mathrm{E} Y_{1: m-1}\right)+D_{n}(k, r),
$$

where

$$
C_{n}(i, k, r):=\left(\begin{array}{c}
n \\
i
\end{array}\right)\left(\begin{array}{c}
r-2-(n-k) \\
r-1-i
\end{array}\right), \quad 1 \leq i \leq m-1,
$$

and

$$
D_{n}(k, r):=\sum_{i=n+1-k}^{m-2} \frac{C_{n}(i, k, r)}{C_{n}(m-1, k, r)}\left(\mathrm{E} X_{1: i}-\mathrm{E} Y_{1: i}\right) .
$$

It is easy to check that

$$
\sup _{n+2-m \leq k \leq n} D_{n}(k, r) \longrightarrow 0 \quad \text { as } n \rightarrow \infty .
$$

This fact, together with (7), (14), and (15), implies the existence of an $n_{0} \geq 1$ satisfying the requirements of part (b) of the lemma.

We are now in a position to show the main results in this section.

Theorem 3. Let $(X, F)$ and $(Y, G)$ be given. For $r \geq 3$, the following assertions are mutually equivalent:

(a) $X \leq_{r} Y$, and there is no $m \in\{2, \ldots, r-1\}$ such that $F_{m}^{-1}(1)>G_{m}^{-1}(1)$, and $F_{i}^{-1}(1)=$ $G_{i}^{-1}(1)$ for $i=m+1, \ldots, r$;

(b) there exists an integer $n_{0} \geq 1$ such that $\boldsymbol{\mu}_{n}^{X} \prec_{r} \boldsymbol{\mu}_{n}^{Y}$ for all $n \geq n_{0}$;

(c) $\boldsymbol{\mu}_{n}^{X} \prec_{r} \boldsymbol{\mu}_{n}^{Y}$ for infinitely many values of $n$.

Proof. Fix $r \geq 3$. Assume that (a) holds true. By Theorem 2, $X \leq_{r} Y$ implies that

$$
s_{k: n}^{[r]}\left(\boldsymbol{\mu}_{n}^{X}\right)-s_{k: n}^{[r]}\left(\boldsymbol{\mu}_{n}^{Y}\right) \leq 0, \quad n \geq r-1,1 \leq k \leq n-(r-2),
$$

and (with the notation in Lemma 6) the additional assumption of (a) ensures that $M=\varnothing$ or $M \neq \varnothing$ and $F_{m}^{-1}(1)-G_{m}^{-1}(1)<0$. In any case, from (16) and Lemma 6, we conclude that (b) holds true. Assertion (b) trivially implies (c). Assume, finally, that (c) holds true. By Theorem 2 we have $X \leq_{r} Y$. Moreover, if there exists $m \in\{2, \ldots, r-1\}$ such that $F_{m}^{-1}(1)>G_{m}^{-1}(1)$, and $F_{i}^{-1}(1)=\bar{G}_{i}^{-1}(1)$ for $i=m+1, \ldots, r$, we find, by Lemma 6 , that there exists an integer $n_{0}$ such that $s_{k: n}^{[r]}\left(\boldsymbol{\mu}_{n}^{X}\right)-s_{k: n}^{[r]}\left(\boldsymbol{\mu}_{n}^{Y}\right)>0$ for all $n \geq n_{0}$ and $k>n-(r-2)$, which is in contradiction with (c). Therefore, (a) holds true, and the proof is complete. 
The next corollary gives the same result as the previous theorem, but only conditions on $F_{r}^{-1}-G_{r}^{-1}$ are required to guarantee the majorization $\boldsymbol{\mu}_{n}^{X} \prec_{r} \boldsymbol{\mu}_{n}^{Y}$ for large enough $n$.

Corollary 1. Let $r \geq 3$, and let $(X, F)$ and $(Y, G)$ be such that $X \leq_{r} Y$ and $F_{r}^{-1}(1)<G_{r}^{-1}(1)$. Then, there exists an integer $n_{0} \geq 1$ such that $\boldsymbol{\mu}_{n}^{X} \prec_{r} \boldsymbol{\mu}_{n}^{Y}$ for all $n \geq n_{0}$.

Examples 1 and 2 show that, when $r \geq 3$, the $r$-ISD does not guarantee the weak $r$-majorization of all the vectors of mean order statistics. Furthermore, Theorem 3 prompts that, in order to have $\boldsymbol{\mu}_{n}^{X} \prec_{r} \boldsymbol{\mu}_{n}^{Y}$ for all $n \geq 1$, we need to impose conditions on the sign of $F_{j}^{-1}(1)-G_{j}^{-1}(1)$ for $2 \leq j \leq r-1$, in addition to the $r$-ISD. This suggests the introduction of the following notion.

Definition 3. Let $r \geq 3$. We say that $Y$ dominates $X$ in the positive $r$-ISD, written $X \leq_{r}^{+} Y$, if $F_{r}^{-1}(t) \leq G_{r}^{-1}(t)$ for all $t \in(0,1)$ and $F_{j}^{-1}(1) \leq G_{j}^{-1}(1)$ for all $2 \leq j \leq r-1$.

Remark 7. From (7), it is clear that (for $r \geq 3$ ) the following assertions are equivalent:

(a) $X \leq_{r}^{+} Y$;

(b) $X \leq_{r} Y$ and $\mathrm{E} X_{1: j} \leq \mathrm{E} Y_{1: j}$ for $1 \leq j \leq r-2$.

If $X$ and $Y$ measure the income level in two populations, the following theorem states that, when $X \leq_{r}^{+} Y$, the expected income in ordered samples from $Y$ (of any size) is more even than that from $X$, according to the weak $r$-majorization. The relations ' $\leq_{r}^{+}$' $(r \geq 3)$ arrange variables by focusing on the left tail of the distributions (the lowest social strata of the populations). The higher $r$ is, the more important the lowest part of the distributions. This suggests that it would be reasonable to consider the relations ' $\leq_{r}^{+}$' (instead of ' $\leq_{r}$ ') as the natural extensions of the first two stochastic dominance rules, ' $\leq_{1}$ ' and ' $\leq_{2}$ '.

Theorem 4. Let $(X, F)$ and $(Y, G)$ be given. For $r \geq 3$, the following assertions are equivalent:

(a) $X \leq_{r}^{+} Y$;

(b) $\boldsymbol{\mu}_{n}^{X} \prec_{r} \boldsymbol{\mu}_{n}^{Y}$ for all $n \geq 1$.

Proof. In view of Remark 7, the conclusion easily follows from Theorem 2, Lemma 5, and the fact that we have $s_{1: n}^{[r]}\left(\boldsymbol{\mu}_{n}^{X}\right)=\mathrm{E} X_{1: n}$ and $s_{1: n}^{[r]}\left(\boldsymbol{\mu}_{n}^{Y}\right)=\mathrm{E} Y_{1: n}$ for $1 \leq n \leq r-2$.

\section{Strong $r$-ISD and $r$-majorization}

In this section we introduce a new stochastic order called strong $r$-ISD. The point (which motivates this introduction) is that such an ordering can be characterized in terms of the $r$-majorization of the vectors of mean order statistics, the $r$-majorization being a new notion (introduced below) that extends the classical reverse majorization of Hardy, Littlewood and Pòlya. The characterization result (Theorem 5, below) then becomes a generalization of Corollary 2.1 of de la Cal and Cárcamo (2006).

Definition 4. Given $(X, F),(Y, G)$, and $r \geq 2$, we say that $X$ is dominated by $Y$ in the strong $r$-ISD, written $X \leq_{r}^{*} Y$, if $F_{r}^{-1}(t) \leq G_{r}^{-1}(t)$ for all $t \in(0,1)$ and $F_{j}^{-1}(1)=G_{j}^{-1}(1)$ for all $2 \leq j \leq r$. 
Remark 8. It is readily checked that (for $r \geq 2$ ) the following assertions are equivalent:

(a) $X \leq_{r}^{*} Y$;

(b) $X \leq_{r} Y$ and $\mathrm{E} X_{1: j}=\mathrm{E} Y_{1: j}$ for $1 \leq j \leq r-1$.

Remark 9. The order ' $\leq_{2}^{*}$ ' is the so-called concave order (see, for instance, Müller and Stoyan (2002, Chapter 1.5) or Shaked and Shanthikumar (2006, Chapter 3.A)), since $X \leq_{2}^{*} Y$ is equivalent to $\mathrm{E} \phi(X) \leq \mathrm{E} \phi(Y)$ for all real concave functions $\phi$ for which the previous expectations are well defined. Thus, the relation ' $\leq_{r}^{*}$ ' $(r \geq 2)$ can be viewed as an extension of the concave order. (A different extension of the concave order can be found in Denuit et al. (1998).)

Next, we give the definition of $r$-majorization of $n$-dimensional vectors. Unlike the weak $r$-majorization, the notion of $r$-majorization requires the equality of the last accumulated sums of their components.

Definition 5. Let $\boldsymbol{x}, \boldsymbol{y} \in \mathbb{R}^{n}$ and $r \geq 2$. We say that $\boldsymbol{x}$ is $r$-majorized by $\boldsymbol{y}$, written $\boldsymbol{x} \prec_{r}^{*} \boldsymbol{y}$, if

$$
\boldsymbol{x} \prec_{r} \boldsymbol{y} \quad \text { and } \quad s_{n: n}^{[j]}(\boldsymbol{x})=s_{n: n}^{[j]}(\boldsymbol{y}), \quad 2 \leq j \leq r .
$$

Remark 10. The expression $\boldsymbol{x} \prec 2$ classical majorization of Hardy, Littlewood and Pòlya (see Marshall and Olkin (1979)). Thus, relation ' $\prec_{r}^{*}$ ' is an extension of the reverse majorization.

Remark 11. When $n<r$, relation ' $\prec_{r}^{*}$ ' becomes trivial, in the following sense: if

$$
\left(x_{1}, \ldots, x_{n}\right)=: \boldsymbol{x} \prec_{r}^{*} \boldsymbol{y}:=\left(y_{1}, \ldots, y_{n}\right)
$$

then $x_{i: n}=y_{i: n}$ for all $1 \leq i \leq n$ (i.e. the increasing rearrangements of $\boldsymbol{x}$ and $\boldsymbol{y}$ are equal). We actually have

$$
s_{n: n}^{[j]}(\boldsymbol{x})=s_{n: n}^{[j]}(\boldsymbol{y}), \quad j=2, \ldots, n+1 .
$$

Thus,

$$
s_{n: n}^{[j]}(\boldsymbol{x})-s_{n: n}^{[j-1]}(\boldsymbol{x})=s_{n: n}^{[j]}(\boldsymbol{y})-s_{n: n}^{[j-1]}(\boldsymbol{y}), \quad j=3, \ldots, n+1,
$$

which is equivalent to

$$
s_{n-1: n}^{[j]}(\boldsymbol{x})=s_{n-1: n}^{[j]}(\boldsymbol{y}), \quad j=3, \ldots, n+1 .
$$

After $n-1$ steps, we obtain

$$
s_{1: n}^{[n+1]}(\boldsymbol{x})=x_{1: n}=y_{1: n}=s_{1: n}^{[n+1]}(\boldsymbol{y}) .
$$

The same argument can now be repeated with the vectors $\left(x_{2: n}, \ldots, x_{n: n}\right)$ and $\left(y_{2: n}, \ldots, y_{n: n}\right)$ to obtain $x_{2: n}=y_{2: n}$. Finally, after $n$ repetitions, we obtain $x_{i: n}=y_{i: n}$ for all $i=1, \ldots, n$.

Remark 12. Some illustrative examples of $r$-majorization of vectors are the following:

$$
\begin{aligned}
& (0.5,1,2) \prec_{3}^{*}(0.6,0.8,2.1), \\
& (0.5,1,1.5,2) \prec_{3}^{*}(0.6,1,1.2,2.2) \text {, } \\
& (0.5,1,1.2,2) \prec_{4}^{*}(0.6,0.7,1.5,1.9) \text {. }
\end{aligned}
$$


The announced characterization of the strong $r$-ISD is stated as follows.

Theorem 5. Let $(X, F)$ and $(Y, G)$ be given. For $r \geq 2$, the following assertions are mutually equivalent:

(a) $X \leq_{r}^{*} Y$;

(b) $\boldsymbol{\mu}_{n}^{X} \prec_{r}^{*} \boldsymbol{\mu}_{n}^{Y}$ for all $n \geq 1$;

(c) $\boldsymbol{\mu}_{n}^{X} \prec_{r}^{*} \boldsymbol{\mu}_{n}^{Y}$ for infinitely many values of $n$.

Proof. The fact that (a) implies (b) directly follows from Theorems 1 and 2, and Lemma 5. Assertion (b) trivially implies (c). Assume, finally, that (c) holds true. From Theorems 1 and 2, we have $X \leq_{r} Y$. Moreover, since $\boldsymbol{\mu}_{n}^{X} \prec_{r}^{*} \boldsymbol{\mu}_{n}^{Y}$ for some $n \geq r$, we obtain

$$
s_{n: n}^{[j]}\left(\boldsymbol{\mu}_{n}^{X}\right)=s_{n: n}^{[j]}\left(\boldsymbol{\mu}_{n}^{Y}\right)
$$

for $2 \leq j \leq r$, and, from Lemma 5 , it is easy to see that such a system of equations implies that $\mathrm{E} X_{1: j}=\mathrm{E} Y_{1: j}$ for $1 \leq j \leq r-1$. We hence conclude that $X \leq_{r}^{*} Y$. This completes the proof.

\section{A generalization of the S-Gini indices}

To provide a better interpretation of the ideas in this section, assume that $(X, F)$ represents the income level of a population, with $\mathrm{E} X \neq 0$. The $S$-Gini indices of $X$ (see Donaldson and Weymark (1980), (1983), Kakwani (1980), and Yitzhaki (1983)) are defined by

$$
G_{n}(X):=1-\frac{\mathrm{E} X_{1: n}}{|\mathrm{E} X|}, \quad n \geq 2 .
$$

In particular, $G_{2}(X)$ is the popular Gini index. It is clear from (17) that $G_{n}(X)$ gives information about the (normalized) difference between the mean income of the population and the expected income of the poorest individual in a random sample of size $n$. For the relevant role of these indices in the analysis of social inequality, poverty, and welfare, we refer the reader to Barrett and Donald (2009) and the references therein.

From Theorem 2, it is clear that the S-Gini indices relate to the $r$-ISD in the following way. If $X \leq_{r} Y$ and $\mathrm{E} X=\mathrm{E} Y(\neq 0)$, then $G_{n}(X) \geq G_{n}(Y)$ for all $n \geq r-1$. In particular, the third inverse dominance is coherent with the Gini index (a result previously obtained in Muliere and Scarsini (1989)).

Theorem 2 also shows that the $r$-ISD is closely connected to the cumulative sums $s_{k: n}^{[r]}\left(\boldsymbol{\mu}_{n}^{X}\right)$, which (by (2)) can be expressed in the form

$$
s_{k: n}^{[r]}\left(\mu_{n}^{X}\right)=\sum_{i=1}^{k}\left(\begin{array}{c}
k-i+r-2 \\
r-2
\end{array}\right) \text { E } X_{i: n}, \quad 1 \leq k \leq n, r \geq 2 .
$$

This suggests the introduction of the following new indices. Given $(X, F), r \geq 2, n \geq 1$, and $1 \leq k \leq n$, we define the absolute indices $\Psi_{k, n}^{[r]}(X)$ by

$$
\Psi_{k, n}^{[r]}(X):=\frac{(r-1) !(k-1) !}{(k+r-2) !} \sum_{i=1}^{k}\left(\begin{array}{c}
k-i+r-2 \\
r-2
\end{array}\right) \text { E } X_{i: n} .
$$


When $X$ describes the income level of a population, $\Psi_{k, n}^{[r]}(X)$ is a weighted mean of the expected incomes of the $k$ poorest individuals in a random sample of size $n$. The greater $r$ is, the more weight is placed on the expected incomes of the poorest. Also, the greater $k$ is, the greater the proportion of poor people in the sample. From Theorem 2 (together with (2)) we have $X \leq_{r} Y$ if and only if

$$
\Psi_{k, n}^{[r]}(X) \leq \Psi_{k, n}^{[r]}(Y)
$$

for all $n \geq r-1$ and $1 \leq k \leq n-(r-2)$. Furthermore, Theorem 4 states that $X \leq_{r}^{+} Y$ if and only if

$$
\Psi_{k, n}^{[r]}(X) \leq \Psi_{k, n}^{[r]}(Y)
$$

for all $n \geq 1$ and $1 \leq k \leq n$. Note also that by (3) we have $\Psi_{k, n}^{[r]}(c)=c$, whenever $c$ is constant. In particular, since $X \leq_{r}^{+} \mathrm{E} X$, we always have $\Psi_{k, n}^{[r]}(X) \leq \mathrm{E} X$.

On the other hand, provided that $\mathrm{E} X \neq 0$, we can normalize the quantity $\Psi_{k, n}^{[r]}(X)$ to obtain the relative indices $J_{k, n}^{[r]}(X)$ given by

$$
J_{k, n}^{[r]}(X):=1-\frac{\Psi_{k, n}^{[r]}(X)}{|\mathrm{E} X|},
$$

which can be viewed as extensions of the S-Gini indices, since $J_{1, n}^{[r]}(X)=G_{n}(X)(r \geq 2)$. Next, we list some properties of these indices. In the statements we assume that $X$ and $Y$ are nonnegative random variables, with $\mathrm{E} X=\mathrm{E} Y>0$, and that $c$ is a positive constant. They all are easy to show, and so we omit the details.

- $J_{k, n}^{[r]}(X) \in[0,1)$.

- $J_{k, n}^{[r]}(X)$ is scale free, that is, $J_{k, n}^{[r]}(c X)=J_{k, n}^{[r]}(X)$.

- $J_{k, n}^{[r]}(c)=0$.

- $X \leq_{r} Y$ if and only if $J_{k, n}^{[r]}(Y) \leq J_{k, n}^{[r]}(X)$ for $n \geq r-1$ and $1 \leq k \leq n-(r-2)$.

- $X \leq_{r}^{+} Y$ if and only if $J_{k, n}^{[r]}(Y) \leq J_{k, n}^{[r]}(X)$ for $n \geq 1$ and $1 \leq k \leq n$.

When $X$ describes the income level of a population, the interpretation is clear: the closer to 0 or 1 the quantity $J_{k, n}^{[r]}(X)$ is, the more even or, respectively, unfair the distribution of the income of $X$ is.

\section{Concluding remarks}

In this paper, the connection between the inverse dominance of degree $r$ ( $r$-ISD) and the mean order statistics was discussed in depth. We showed that the $r$-ISD between two variables is intrinsically linked with the weak $r$-majorization of the corresponding vectors of expected order statistics, where the weak $r$-majorization is a new concept introduced in this work, which generalizes the classical notion of reverse weak majorization of Hardy, Littlewood and Pòlya. The weak $r$-majorization orders vectors by focusing on their smallest components: the larger $r$ is, the more importance is given to the smallest components.

On the other hand, the results clearly showed that the cases $r=1,2$ (which correspond to the usual dominance of first and second order) differ substantially from those with $r \geq 3$, and lead in a natural way to the introduction of two new stochastic orderings, called positive $r$-ISD and strong $r$-ISD. These new relations can be viewed as natural extensions of the first two dominance rules or concave-type stochastic orders, and have a clear economic interpretation 
that make them appropriate to compare the social inequality and the distribution of wealth in two populations.

Finally, our main results also showed that all the information contained in the $r$-ISD of two variables can be given in terms of a family of inequality indices that extends the well-known family of S-Gini indices. We believe these ideas contribute to a better understanding of the meaning of the $r$-ISD.

\section{Acknowledgements}

The authors thank the anonymous referee for valuable comments and suggestions that improved the paper and for pointing out references Donaldson and Weymark (1980), (1983) regarding the S-Gini indices.

This research was supported by the Spanish MEC, grant number MTM2008-06281-C02-02.

\section{References}

Aaberge, R. (2009). Ranking intersecting Lorenz curves. Soc. Choice Welf. 33, 235-259.

Arnold, B. C., Balakrishnan, N. and Nagaraja, H. N. (2008). A First Course in Order Statistics. Society for Industrial and Applied Mathematics, Philadelphia, PA.

Baíllo, A., Berrendero, J. R. And CárCAmo, J. (2009). Tests for zero-inflation and overdispersion: a new approach based on the stochastic convex order. Comput. Statist. Data Anal. 53, 2628-2639.

Barrett, G. F. and Donald, S. G. (2009). Statistical inference with generalized Gini indices of inequality, poverty, and welfare. J. Business Econom. Statist. 27, 1-17.

Berrendero, J. R. AND CÁrCAMo, J. (2009). Characterizations of exponentiality within the HNBUE class and related tests. J. Statist. Planning Infer. 139, 2399-2406.

Berrendero, J. R. and Cárcamo, J. (2010). Tests for the second order stochastic dominance based on L-statistics. To appear in J. Business Econom. Statist.

De la Cal, J. and Cárcamo, J. (2006). Stochastic orders and majorization of mean order statistics. J. Appl. Prob. 43, 704-712.

Dentcheva, D. And Ruszczynski, A. (2006). Inverse stochastic dominance constraints and rank dependent expected utility theory. Math. Program. 108, 297-311.

Denuit, M., LeFÈvRe, C. AND ShaKed, M. (1998). The $s$-convex orders among real random variables, with applications. Math. Inequal. Appl. 1, 585-613.

Denuit, M., Lefèvre, C. And Shaked, M. (2000). On the theory of high convexity stochastic orders. Statist. Prob. Lett. 47, 287-293.

Denuit, M., Dhaene, J., Goovaerts, M. And KaAs, R. (2005). Actuarial Theory for Dependent Risks. John Wiley, Chichester.

Donaldson, D. and Weymark, J. A. (1980). A single-parameter generalization of the Gini indices of inequality. J. Econom. Theory 22, 67-86.

Donaldson, D. and Weymark, J. A. (1983). Ethical flexible indices for income distributions in the continuum. J. Econom. Theory 29, 353-358.

Fishburn, P. C. (1976). Continua of stochastic dominance relations for bounded probability distributions. J. Math. Econom. 3, 295-311.

HuAng, J. S. (1989). Moment problem of order statistics: a review. Internat. Statist. Rev. 57, 59-66.

Johnson, N. L., Kemp, A. W. And Kotz, S. (2005). Univariate Discrete Distributions, 3rd edn. John Wiley, Hoboken, NJ.

KAKWANI, N. (1980). On a class of poverty measures. Econometrica 48, 437-446.

LAI, C.-D. AND XIE, M. (2006). Stochastic Ageing and Dependence for Reliability. Springer, Berlin.

LAmbert, P. J. (1993). The Distribution and Redistribution of Income, a Mathematical Analysis, 2nd edn. BasilBlackwell, Oxford.

LEVY, H. (1992). Stochastic dominance and expected utility: survey and analysis. Manag. Sci. 38, 555-593.

Maccheroni, F., Muliere, P. And Zoli, C. (2005). Inverse stochastic orders and generalized Gini functionals. Metron 53, 529-559.

Marshall, A. W. And Olkin, I. (1979). Inequalities: Theory of Majorization and Its Applications. Academic Press, New York.

Muliere, P. AND Scarsini, M. (1989). A note on stochastic dominance and inequality measures. J. Econom. Theory 49, 314-323. 
Müller, A. And Stoyan, D. (2002). Comparison Methods for Stochastic Models and Risks. John Wiley, Chichester. Shaked, M. And Shanthikumar, J. G. (2006). Stochastic Orders. Springer, New York.

Shorrocks, A. F. AND Foster, J. E. (1987). Transfer sensitive inequality measures. Rev. Econom. Stud. 54, $485-497$. WANG, S. S. AND Young, V. R. (1998). Ordering risks: expected utility theory versus Yaari's dual theory of risk. Insurance Math. Econom. 22, 145-161.

YAARI, M. E. (1987). The dual theory of choice under risk. Econometrica 55, 95-115.

YITZHAKI, S. (1983). On the extension of the Gini inequality index. Internat. Econom. Rev. 24, 617-628.

ZoLI, C. (1999). Intersecting generalized Lorenz curves and the Gini index. Soc. Choice Welf. 16, 183-196.

ZoLI, C. (2002). Inverse stochastic dominance, inequality measurement and Gini indices. In Inequalities: Theory, Measurement and Applications (Bordeaux, 2000), Springer, Vienna, pp. 119-161. 